\section{Quality of life and function after electroconvulsive}

\section{therapy}

W. VAUGHN McCALL, AARON DUNN and PETER B. ROSENQUIST
Electroconvulsive therapy (ECT) is widely acknowledged as an effective and appropriate acute treatment for major depressive illness (American Psychiatric Association Committee on Electroconvulsive Therapy, 2001; UK ECT Review Group, 2003). However, questions remain regarding the extent and longevity of the effects of this therapy on memory, and whether ECT is associated with a net improvement in function and quality of life. Correspondingly, guidance on the use of ECT in the UK issued by the National Institute for Clinical Excellence (NICE) recommended sharp restrictions on the use of this therapy until more information becomes available about its effects on memory, quality of life and other pertinent health outcomes (National Institute for Clinical Excellence, 2003). The NICE guidance states that 'no study adequately captured... quality of life' and that 'better quality-of-life information is needed for people considered for, or who have received, ECT' (National Institute for Clinical Excellence, 2003: paras 4.1.2, 5.5).

Indeed, the literature on mood and cognition has generally shown that poorer cognitive status is associated with worse quality of life in patients with depression. These relationships are true whether the sample of interest is patients with depression secondary to dementia (Pearson et al, 1989) or patients with primary depression and secondary cognitive dysfunction (McCall \& Dunn, 2003). These observations suggest that the cognitive side-effects of ECT should limit any beneficial antidepressant effect on quality of life. However, we and others have previously shown that ECT results in a net improvement in function and quality of life for a period of at least 1 year after treatment (McCall et al, 2001; Casey et al, 1996). We have therefore examined the simultaneous, respective contributions of antidepressant efficacy and cognitive side-effects to the changes in function and quality of life in patients given ECT. We began with the hypothesis that antidepressant efficacy would be associated with improvement in function and quality of life, whereas cognitive side-effects would be associated with a dampening of function and quality of life in patients treated with ECT.

\section{METHOD}

\section{Study sample}

The sample was recruited from patients with major depressive episode diagnosed on the basis of a structured interview (Ventura et al, 1998). Written informed consent was obtained after a complete description of the study had been given to the participants, and the protocol was approved by the local institutional review board. Participants were at least 18 years old, with no history of schizophrenia or schizoaffective disorder, active substance misuse, learning disability or neurological illness; their Mini-Mental State Examination (MMSE; Folstein et al, 1975) scores were 18 or over. No patient had received ECT during the previous 4 months. Baseline scores on the 21-item Hamilton Rating Scale for Depression (HRSD; Hamilton, 1960) were 20 or over. The adequacy of antidepressant drug therapy during the current major depressive episode was determined using a dichotomised rating scale (adequate treatment $v$. inadequate treatment) (Prudic et al, 1996; McCall et al, 1999a; Sackeim, 2001). Antidepressants, anticonvulsants and lithium were discontinued prior to the first ECT session and benzodiazepines were tapered to $4 \mathrm{mg}$ or less per day of lorazepam or its equivalent. Participants received open treatment with antidepressant medication during the month-long period of observation after ECT, with the choice of medication determined by the attending physician.

\section{Procedure}

Right unilateral electrode placement followed the d'Elia position, whereas bilateral placement was in the frontotemporal position (D'Elia \& Raotma, 1975). Electrode placement was randomly assigned. A MECTA SRI device (MECTA Corp., Tualatin, Oregon, USA) delivered the constant current, bidirectional brief pulse. Seizure threshold was estimated in all patients at the first therapy session, with an approximately $50 \%$ increment change at each subsequent session. Patients with right 
unilateral placement received a stimulus 8 times their initial seizure threshold at the second and subsequent treatment sessions, while patients in the bilateral placement group received a stimulus 1.5 times their seizure threshold at the second and subsequent treatment sessions. Additional details of ECT technique can be found elsewhere (McCall et al, 2002a). The total number of treatments was determined by the ward psychiatrist, who was masked to the patients' randomisation status.

\section{Depression severity and response criteria}

Patients provided a self-report of depression severity with the Beck Depression Inventory (BDI; Beck et al, 1988), and a trained rater masked to patient status measured depression severity with the 21-item HRSD following a semi-structured interview. The HRSD was completed 1-3 days prior to the first ECT session, $24 \mathrm{~h}$ after each mid-course session, 1-2 days after the last session, and 2 weeks and 4 weeks after the last session. The BDI was completed before and immediately after the course of therapy, and at 2 weeks and 4 weeks after the last session. Antidepressant response was defined as a decrease of at least $60 \%$ in HRSD scores and a final score of 12 or less after completion of the therapy. Relapse among the responders was defined as a $50 \%$ increase in HRSD scores from the end of therapy and an HRSD score of 14 or more at both the 2week and the 4-week intervals.

\section{Cognitive assessment}

Cognition was assessed 1-3 days prior to ECT, 1-2 days after the course of ECT and at 2 and 4 weeks after ECT by a masked rater. Global cognition was evaluated with the MMSE. Retrograde autobiographical memory was measured with the Personal Memory Questionnaire (McElhiney et al, 1995). Anterograde amnesia for verbal memory function was assessed with the Rey Auditory Verbal Learning Test (Ryan et al, 1986) with a 20 min delay (RAVLT-DR). Anterograde amnesia for figural memory function was tested with the Rey Complex Figure Test (Recall Phase) with a 20 min delay (RCFT-DR; Spreen $\&$ Strauss, 1991).

\section{Function and quality of life}

Function was measured with an instrumental activities of daily living (IADL) scale and an activities of daily living scale, the Personal Self-Maintenance Scale (PSMS; Lawton \& Brody, 1969). The IADL scale has been shown to be sensitive to change in this sample and to demonstrate good reliability between patients and their caregivers, with a correlation of 0.95 for the change scores (McCall et al, 2002b). Quality of life was assessed with the Daily Living and Role Functioning (DLRF) sub-scale and the Relationship to Self and Others (RSO) sub-scale of the Basis-32 instrument (Eisen et al, 1994).

\section{Statistics}

Means are reported with standard deviations. The means of continuous variables were compared with $t$ tests or adjusted analyses of variance. Frequency distributions were compared with the $\chi^{2}$ statistic. Models of the change in function and quality of life were created for the 2-week and 4-week post-ECT time points, compared with the pre-ECT baseline. A total of eight linear regression models were created (DLRF, RSO, IADL and PSMS scores at both 2 weeks and 4 weeks). All measurements within the models were expressed as the percentage change from baseline, with the exception of age and gender. Predictor variables were initially handled as block variables. Age and gender were grouped as the block variable 'demographic', the BDI and HRSD scores were grouped as the block variable 'mood' and the MMSE, RAVLT-DR, RCFT-DR and autobiographical memory measures were grouped as 'cognitive'. The models all fit the general formula (Function/QOL) $=$ $\left(\alpha+\beta_{1}\right.$ demographic $+\beta_{2}$ mood $+\beta_{3}$ cognitive $)$. If the overall model was significant, we then examined which of the block variables was significant. Finally, only if a block variable was significant did we examine the component variables of the block to test their significance within the full model and within univariate regressions. Statistical significance was accepted for $\alpha$ set at 0.05. All tests were two-sided.

\section{RESULTS}

Participants allocated to bilateral or right unilateral electrode placement were similar, and the groups were not significantly different in age, gender, educational attainment, adequacy of antidepressant medication therapy at all time points, pre-treatment severity of depressive symptoms, duration
Table I Demographic and clinical features of the sample

\begin{tabular}{lc}
\hline Age, years: mean (s.d.) & $57.3(16.4)$ \\
Gender (male/female), $n / n$ & $49 / 28$ \\
Educational attainment, years: & $12.7(3.5)$ \\
mean (s.d.) & \\
In-patients/out-patients, $n / n$ & $71 / 6$ \\
Electrode placement & \\
$\quad$ Right unilateral, $n$ & 40 \\
$\quad$ Bilateral, $n$ & 37 \\
Duration of index MDE, weeks: & $25.0(20.3)$ \\
mean (s.d.) & \\
Number of prior MDEs: mean (s.d.) & $2.6(1.7)$ \\
Age at onset of first MDE, years: & $37.9(17.6)$ \\
mean (s.d.) & \\
\hline
\end{tabular}

MDE, major depressive episode.

of the current episode, number of prior depressive episodes and age at onset of the first depressive episode. Similarly, there was no statistically or clinically significant between-group difference for antidepressant response or cognitive side-effects at any time point (McCall et al, 2002a; Table $1)$. We therefore pooled the results from both groups for all subsequent analysis. In general, prior to ECT, participants were severely depressed, had minimal cognitive dysfunction and had significant deficits in quality of life and functional status (Table 2).

Sixty-two patients $(81 \%)$ met our definition of 'adequate treatment' prior to ECT, and $39(55 \%)$ and $47(70 \%)$ met the definition at 2 weeks and 4 weeks, respectively (Prudic et al, 1996). Prescribed antidepressant regimens at 2 weeks included selective serotonin reuptake inhibitors (17 patients); venlafaxine (13 patients), atypical antidepressants (bupropion, mirtazapine or nefazodone; 22 patients), tricyclic antidepressants (12 patients, including 3 on lithium) and monoamine oxidase inhibitors (2 patients). The antidepressant regimens at 4 weeks were similar.

Forty-one participants from the full sample of 77 patients had complete data for every variable at every time point. Missing data from the other 36 patients were assumed to be missing at random; therefore imputation techniques were not employed. Pre-post difference scores and regression models were examined for both all available data (full data-set) and for the 41 participants with a complete data-set. Statistical inference was the same for both 
Table 2 Changes in mood, cognition, quality of life and function for the full data-set

\begin{tabular}{|c|c|c|c|c|c|c|c|c|c|}
\hline \multirow[t]{3}{*}{ Measure } & \multicolumn{9}{|c|}{ Change from baseline } \\
\hline & \multicolumn{3}{|c|}{ Baseline scores } & \multicolumn{3}{|c|}{2 weeks after ECT (\%) } & \multicolumn{3}{|c|}{4 weeks after ECT (\%) } \\
\hline & Mean & (s.d.) & $n$ & Mean & (s.d.) & $n^{\prime}$ & Mean & (s.d.) & $n^{\prime}$ \\
\hline $\mathrm{HRSD}^{2}$ & 28.9 & $(4.9)$ & 77 & -44.1 & $(26.7)^{* * *}$ & 70 & -45.7 & $(29.4)^{* * *}$ & 61 \\
\hline $\mathrm{BDI}^{2}$ & 34.1 & $(10.7)$ & 74 & -40.9 & $(76.1)^{* * *}$ & 69 & -47.2 & $(54.2)^{* * *}$ & 66 \\
\hline $\mathrm{MMSE}^{3}$ & 27.4 & $(2.9)$ & 77 & 3.2 & $(10.8)^{*}$ & 69 & 4.9 & $(I I .2)^{* *}$ & 59 \\
\hline RAVLT-DR ${ }^{4}$ & 6.4 & $(4.0)$ & 74 & 40.1 & $(160.7)$ & 62 & 48.8 & $(175.8)$ & 53 \\
\hline RCFT-DR ${ }^{4}$ & 13.7 & (8.7) & 71 & 52.9 & $(123.1)^{* * *}$ & 56 & 65.9 & $(124.8)^{* * *}$ & 49 \\
\hline Autobiographical & 47.0 & $(15.1)$ & 75 & -20.0 & $(12.6)^{* * *}$ & 70 & -15.0 & $(13.1)^{* * *}$ & 66 \\
\hline $\mathrm{DLRF}^{2}$ & 3.0 & $(0.7)$ & 75 & -46.8 & $(33.9)^{* * *}$ & 70 & -52.4 & $(39.9)^{* * *}$ & 67 \\
\hline $\mathrm{RSO}^{2}$ & 2.3 & $(1.0)$ & 75 & -46.1 & $(58.9)^{* * *}$ & 70 & -52.4 & $(44.3)^{* * *}$ & 67 \\
\hline $\mathrm{IADL}^{3}$ & 20.2 & $(5.1)$ & 76 & 17.8 & $(33.3)^{* * *}$ & 70 & 29.0 & $(38.9)^{* * *}$ & 67 \\
\hline PSMS $^{3}$ & 27.4 & (2.7) & 76 & 5.6 & $(14.2)^{* *}$ & 71 & 5.6 & $(14.0)^{* *}$ & 67 \\
\hline
\end{tabular}

BDI, Beck Depression Inventory; DLRF, Daily Living and Role Function sub-scale; ECT, electroconvulsive therapy; HRSD, Hamilton Rating Scale for Depression; IADL, Instrumental Activities of Daily Living; MMSE, Mini-Mental State Examination; PSMS, Personal Self-Maintenance Scale; RAVLT-DR, Rey Auditory Verbal Learning Test, Delayed Recall; RCFT-DR, Rey Complex Figure Test, Delayed Recall; RSO, Relationship to Self and Others sub-scale.

I. Number of pairs available.

2. Lower scores are better.

3. Higher scores are better

4. Number of elements recalled after 20 min delay.

5. Number of personal memory items reported.

$* P<0.05, * * P<0.01, * * * P<0.0001$; paired $t$-test follow-up value $v$. baseline.

the full data-set and the complete data-set; therefore, results are presented only for the full data-set.

The immediate post-ECT responder rate was $66 \%$, with $37 \%$ of the responders relapsing within the first month after ECT. The sample showed improvement in every measure of mood, cognition, quality of life and function at both the 2 -week and

Table 3 Determinants of quality of life and function

\begin{tabular}{|c|c|c|c|c|c|}
\hline \multirow[t]{2}{*}{ Model } & \multirow{2}{*}{$\begin{array}{c}\text { Overall } \\
\qquad F\end{array}$} & \multirow[t]{2}{*}{ (d.f.) } & \multicolumn{3}{|c|}{ Partial $F$} \\
\hline & & & $\Delta$ Demographic & $\Delta$ Mood & $\Delta$ Cognitive \\
\hline \multicolumn{6}{|l|}{$\Delta \mathrm{DLRF}$} \\
\hline At 2 weeks & $7.3 * * *$ & 8,43 & 0.3 & $17.3^{* * *}$ & 0.8 \\
\hline At 4 weeks & $7.2^{* * *}$ & 8,33 & 0.3 & $17.4^{* * *}$ & 0.2 \\
\hline \multicolumn{6}{|l|}{$\Delta \mathrm{RSO}$} \\
\hline At 2 weeks & $7.9 * * *$ & 8,43 & 0.4 & $18.6 * * *$ & 0.4 \\
\hline At 4 weeks & $5.4^{* * *}$ & 8,33 & 0.5 & $14.3^{* * *}$ & 0.2 \\
\hline \multicolumn{6}{|l|}{$\triangle \mathrm{IADL}$} \\
\hline At 2 weeks & $3.1^{* *}$ & 8,42 & 2.0 & 1.2 & $2.6^{*}$ \\
\hline At 4 weeks & 1.2 & 8,33 & 0.2 & 0.1 & 1.6 \\
\hline \multicolumn{6}{|l|}{$\triangle \mathrm{PSMS}$} \\
\hline At 2 weeks & I.I & 8,42 & 0.6 & 3.5 & 0.4 \\
\hline At 4 weeks & 0.6 & 8,33 & 0.4 & 0.3 & 0.6 \\
\hline
\end{tabular}

DLRF, Daily Living and Role Function sub-scale; IADL; Instrumental Activities of Daily Living; RSO, Relationship to Self and Others sub-scale; PSMS, Personal Self-Maintenance Scale. the 2-week and 4-week assessments, a few had worse scores. There were 70 patients who had complete scores for baseline and 2-week measures of DLRF, RSO, IADL and PSMS, including 3, 8, 14 and 12 patients who had worse scores on the respective scales. There were 67 patients who had complete scores for baseline and 4-week measures of DLRF, RSO, IADL and PSMS, including 3, 8, 6 and 8 who had worse scores, respectively. Compared with patients with improved quality of life and function, patients with worse quality of life or function scores had lower baseline HRSD and RCFT-DR scores, greater decrements in retrograde memory and less improvement in HRSD score.

All of the models of the change in DLRF and RSO scores were highly significant, and mood was the only significant block variable (Table 3). Fractionation of the 'mood' block revealed that only the HRSD was statistically related to DLRF or RSO when the HRSD and BDI measures were both present in the models. Reductions in depression severity were associated with reductions in deficits in DLRF and RSO.

The significant model of function was IADL at 2 weeks after ECT. 'Cognitive' was the only block variable that was significant within that model. Further fractionation of 'cognitive' showed that only the change in the MMSE score was related to the change in IADL score at 2 weeks $(r=0.3, P<0.05 ; n=67)$. The contribution of the MMSE to the variance of IADL score could not be explained away as a confound of mood, as the change in MMSE score and the change in the HRSD score at 2 weeks were not related ( $r=-0.1, P=0.38 ; n=69)$.

\section{DISCUSSION}

A key finding of this study is that patients' quality of life and function are improved as early as 2 weeks after the completion of ECT. We have previously reported that function and quality of life improve after ECT, and to a greater extent than in medication-treated patients, but in that study the first measurement was taken at 4 weeks after ECT (McCall et al, 2001). The relevance of our findings is underscored by our prior report that function and quality of life measures appear to be key indicators for the use of ECT in depressed patients (McCall et al, 1999b), and that improvement in these domains is 
sustained for up to 1 year in patients treated with ECT (Casey et al, 1996; McCall et al, 2001).

The change in our quality of life measures (DLRF and RSO) was related to change in mood. This is consistent with earlier cross-sectional and longitudinal data which showed that mood, not cognition, was related to these two variables (McCall et al, 1999a, 2001; McCall \& Dunn, 2003). The finding that only the change in HRSD score, not the change in BDI score, was significantly related to change in quality of life when both the HRSD and BDI were in the models may be explained by the use of the HRSD to determine response status to ECT. The decision to continue or stop ECT was in part related to change in the HRSD score, not the BDI score.

Although our sample showed improvement in both measures of function at the 2-week and 4-week time points (Table 2), our models could only predict the change in IADL at 2 weeks. Interestingly, change in IADL at 2 weeks was most closely related to change in cognition, not mood. In particular, improvement in the MMSE, but not any of the specific memory measures, was related to improvement in IADL at 2 weeks. This is consistent with our earlier finding that baseline IADL function in patients treated with ECT is related to the MMSE score but not to mood (McCall \& Dunn, 2003).

Given the extensive research on cognitive deficits related to ECT, it may seem remarkable that our measures of global cognition, anterograde verbal memory and anterograde figural memory showed improvement at 2 weeks and 4 weeks after ECT compared with the pre-therapy baseline. However, cognitive improvement with ECT has been reported repeatedly over the past 40 years (Fink, 1979; Pisvejc et al, 1998). Cognitive problems may be an intrinsic part of depressive illness (Weingartner et al, 1981; King et al, 1991), and it is logical that treatment of depression should be associated with improvement on cognitive tests. Although the improvement on neuropsychological tests could be attributed to a practice effect, it is equally likely that it represents true improvement in cognitive efficiency in patients whose cognitive efficiency had been overtaxed by depression. Similar findings of improved cognition have been reported in stroke patients recovering from depression (Narushima et $a l, 2003)$ and in clinical trials of antidepressant medication in primary depression

\section{CLINICAL IMPLICATIONS}

Quality of life and function are improved within the first month after electroconvulsive therapy in patients with depression.

Improvement in function is related to improved cognition, whereas improved quality of life is related to improved mood.

- A restrictive attitude toward this therapy is not warranted on the basis of its effects on quality of life and function.

\section{LIMITATIONS}

This study was limited by a modest sample size.

The period of follow-up was just I month.

Some data were assumed missing at random.

W. VAUGHN McCALL, MD, MS, AARON DUNN, BA, PETER B. ROSENQUIST, MD, Department of Psychiatry and Behavioral Medicine, Wake Forest University Health Sciences, Winston-Salem, North Carolina, USA

Correspondence: W.Vaughn McCall, Department of Psychiatry and Behavioral Medicine, Wake Forest University Health Sciences, Medical Center Boulevard,Winston-Salem, North Carolina 27I57-I07I, USA. Tel: 3367162911 ; fax: 336 716 3508; e-mail: vmccall@wfubmc.edu

(First received 20 January 2004, final revision 2 June 2004, accepted 26 June 2004)

(Reynolds et al, 1987; Reifler et al, 1989; Newhouse et al, 2000; Cassano et al, 2002).

This study has some limitations. The number of participants was small, and there were some missing data. Still, the results from the 'all available' (full) data-set led to the same inference as did the results of the complete data-set. Patients received naturalistic treatment during follow-up with a wide range of antidepressant medications in various dosages as prophylaxis against relapse. It is unclear how medication status might have affected mood, cognition, quality of life and function. Although the follow-up period was only a month long, the mood, cognitive and neurophysiological effects of ECT fade within weeks, perhaps with the exception of persistent autobiographical memory loss. Although autobiographical memory did not figure into any of our models during the first month of follow-up, it might be more important to assessment of quality of life months after ECT.

In summary, ECT is associated with improvement in quality of life, function, mood, anterograde memory and global cognitive status at 2 weeks and 4 weeks after this therapy. Most patients experienced improvement in quality of life and function. Change in perceived quality of life seems to be most influenced by changes in mood, whereas change in IADL function at 2 weeks was related to change in global cognition. The results are consistent with the premise that ECT produces a net improvement in health for most patients, and should help fill in the knowledge gap that led to the restrictive guidance on the use of ECT in the UK (National Institute for Clinical Excellence, 2003).

\section{ACKNOWLEDGEMENT}

The study was funded by the National Institute of Mental Health, Award MHOI090.

\section{REFERENCES}

American Psychiatric Association Committee on Electroconvulsive Therapy (200I) The Practice of Electroconvulsive Therapy: Recommendations for Treatment, Training and Privileging. Washington, DC: APA.

Beck, A.T., Steer, R. A. \& Garbin, M. G. (1988) Psychometric properties of the Beck Depression Inventory: twenty-five years of evaluation. Clinical Psychology Review, 8, 77-100 
Casey, P., Meagher, D. \& Butler, E. (1996) Personality, functioning, and recovery from major depression. Journal of Nervous and Mental Disease, 184, 240-245.

Cassano, G. B., Puca, F., Scapicchio, P. L., et al (2002) Paroxetine and fluoxetine effects on mood and cognitive functions in depressed nondemented elderly patients. Journal of Clinical Psychiatry, 63, 396-402.

D'Elia, G. \& Raotma, H. (1975) Is unilateral ECT less effective than bilateral ECT? British Journal of Psychiatry 126, 83-89.

Eisen, S. V., Dill, D. L. \& Grob, M. C. (1994) Reliability and validity of a brief patient-report instrument for psychiatric outcome evaluation. Hospital and Community Psychiatry, 45, 242-247.

Fink, M. (1979) Convulsive Therapy. New York: Raven Press.

Folstein, M. F., Folstein, S. E. \& McHugh, P. R. (1975) 'Mini-mental state': a practical method for grading the cognitive state of patients for the clinician. Journal of Psychiatric Research, 12, 189-198.

Hamilton, M. (1960) A rating scale for depression. Journal of Neurology, Neurosurgery and Psychiatry, 23, $56-62$

King, D. A., Caine, E. D., Conwell, Y., et al (199I) The neuropsychology of depression in the elderly: a comparative study of normal aging and Alzheimer's disease. Journal of Neuropsychiatry and Clinical Neurosciences, 3, 163-168.

Lawton, M. P. \& Brody, E. M. (1969) Assessment of older people: self-maintaining and instrumental activities of daily living. Gerontologist, 9, 179-186.

McCall, V. \& Dunn, A. (2003) Cognitive deficits are associated with functional impairment in severely depressed patients. Psychiatry Research, I2I, 179-184.

McCall, W. V., Cohen, W., Reboussin, B., et al (1999a) Effects of mood and age on quality of life in depressed inpatients. Journal of Affective Disorders, 55, 107-II4.
McCall, W. V., Cohen, W., Reboussin, B., et al (1999b) Pretreatment differences in specific symptoms and quality of life among depressed inpatients who do and do not receive electroconvulsive therapy: a hypothesis regarding why the elderly are more likely to receive ECT. Journal of ECT, 15, 193-20|.

McCall, W.V., Reboussin, B. A., Cohen, W., et al (200I) Electroconvulsive therapy is associated with superior symptomatic and functional change in depressed patients after psychiatric hospitalization. Journal of Affective Disorders, 63, 17-25.

McCall, W. V., Dunn, A., Rosenquist, P. B., et al (2002a) Markedly suprathreshold right unilateral ECT versus minimally suprathreshold bilateral ECT: antidepressant and memory effects. Journal of ECT, 18 126-129.

McCall, W. V., Dunn, A. G., Rosenquist, P., et al (2002b) Proxy validation of patient self-reports of ADL and IADL function before and after electroconvulsive therapy. Journal of ECT, 18, 74-79.

McElhiney, M. C., Moody, B. J., Steif, B. L., et al (1995) Autobiographical memory and mood: effects of electroconvulsive therapy. Neuropsychology, 9, 50I-517.

Narushima, K., Chan, K., Kosier, J., et al (2003) Does cognitive recovery after treatment of poststroke depression last? A 2-year follow-up of cognitive function associated with poststroke depression. American Journal of Psychiatry, 160, II57-1162.

National Institute for Clinical Excellence (2003) Guidance on the Use of Electroconvulsive Therapy. London: NICE.

Newhouse, P. A., Krishnan, K. R., Doraiswamy, P. M. et al (2000) A double-blind comparison of sertraline and fluoxetine in depressed elderly outpatients. Journal of Clinical Psychiatry, 6I, 559-568.

Pearson, J. L., Teri, L., Reifler, B. V., et al (1989) Functional status and cognitive impairment in Alzheimer's patients with and without depression. Journal of the American Geriatric Society, 37, |II7-|12।.
Pisvejc, J., Hyrman, V., Sikora, J., et al (1998) A comparison of brief and ultrabrief pulse stimuli in unilateral ECT. Journal of ECT, 14, 68-75.

Prudic, J., Haskett, R. F., Mulsant, B., et al (1996) Resistance to antidepressant medications and shortterm clinical response to ECT. American Journal of Psychiatry, 153, 985-992.

Reifler, B. V., Teri, L., Raskind, M., et al (1989) Double-blind trial of imipramine in Alzheimer's disease patients with and without depression. American Journal of Psychiatry, 146, 45-49.

Reynolds, C. F., Perel, J. M., Kupfer, D. J., et al (1987) Open-trial response to antidepressant treatment in elderly patients with mixed depression and cognitive impairment. Psychiatry Research, 2I, III-122.

Ryan, J. J., Geisser, M. E., Randall, D. M., et al (1986) Alternate form reliability and equivalency of the Rey auditory verbal learning test. Journal of Clinical and Experimental Neuropsychology, 8, 611-616.

Sackeim, H. A. (200I) The definition and meaning of treatment-resistant depression. Journal of Clinical Psychiatry, 62 (suppl. 16), 10-17.

Spreen, O. \& Strauss, E. (1991) A Compendium of Neuropsychological Tests. New York: Oxford University Press.

UK ECT Review Group (2003) Efficacy and safety of electroconvulsive therapy in depressive disorders: a systematic review and meta-analysis. Lancet, 361, 799-808.

Ventura, J., Liberman, R. P., Green, M. F., et al (1998) Training and quality assurance with the Structured Clinical Interview for DSM-IV (SCID-I/P). Psychiatry Research, 79, 163-173.

Weingartner, H., Cohen, R. M., Murphy, D. L., et a (198I) Cognitive processes in depression. Archives of General Psychiatry, 38, 42-47. 